\title{
SÍNTESIS HISTÓRICA Y ESTADO ACTUAL DE LA PESQUERÍA DE VIEIRA PATAGÓNICA Zygochlamys patagonica (KING 1832) EN LA ARGENTINA. BIOLOGÍA, EVALUACIÓN DE BIOMASA Y MANEJO
}

\author{
Silvana Campodónico ${ }^{1}$, Mariana Escolar, Julio García y Aníbal Aubone \\ Instituto Nacional de Investigación y Desarrollo Pesquero (INIDEP), \\ Paseo Victoria Ocampo No 1, Escollera Norte, B7602HSA - Mar del Plata, Argentina \\ ${ }^{1}$ correo electrónico: scampodo@inidep.edu.ar
}

\begin{abstract}
RESUMEN. La vieira patagónica Zygochlamys patagonica (King 1832) es un molusco bivalvo que se distribuye alrededor de América del Sur en el área comprendida entre los $36^{\circ} \mathrm{S}$ en el Océano Atlántico y los $42^{\circ} \mathrm{S}$ en el Océano Pacífico. Las agregaciones más importantes a nivel comercial se encuentran en el Océano Atlántico a lo largo del frente de talud asociadas a la isobata de $100 \mathrm{~m}$. La pesquería de esta especie en la Argentina se inició formalmente en 1996 con el esfuerzo pesquero de cuatro buques factoría arrastreros. En los últimos años el promedio de desembarques anuales se situó alrededor de las 5.000 t con un valor medio de USD 9.300 la tonelada de callo, cifra que la ubicaron entre las cuatro pesquerías más importantes del país. En el presente trabajo se describen los avances recientes en materia de investigación científica a 22 años de su inicio. Se detallan, además, los aspectos más relevantes en lo que respecta a biología, explotación comercial y evolución de las medidas de administración que llevaron a establecer un sistema de manejo adaptable.
\end{abstract}

Palabras clave: Zygochlamys patagonica, vieira patagónica, evaluación de stock, síntesis histórica, biología, manejo pesquero, Argentina.

\section{HISTORICAL SYNTHESIS AND CURRENT STATUS OF PATAGONIAN SCALLOP Zygochlamys patagonica (KING 1832) FISHERY IN ARGENTINA. BIOLOGY, STOCK ASSESSMENT AND MANAGEMENT}

\begin{abstract}
Patagonian scallop Zygochlamys patagonica (King 1832) is a bivalve mollusk that distributes around South America in the area comprised between $36^{\circ} \mathrm{S}$ in the Atlantic Ocean and $42^{\circ} \mathrm{S}$ in the Pacific Ocean. The most important aggregations at commercial level are found in the Atlantic Ocean along the shelf break front associated to the $100 \mathrm{~m}$ isobath. The fishery of this species in Argentina was formally initiated in 1996 with the fishing effort of four trawling factory vessels. In recent years the average annual landings was around 5,000 $\mathrm{t}$ with a mean value of USD 9,300 per ton of meat, figure that placed it among the four most important fisheries in the country. In this paper the recent advances in scientific research at 22 years of its beginning are described. In addition, the most relevant aspects as regards biology, commercial exploitation and evolution of the administration measures that led to establish an adaptable management system are detailed.
\end{abstract}

Key words: Zygochlamys patagonica, Patagonian scallop, stock assessment, historical synthesis, biology, fishery management, Argentina. 


\section{INTRODUCCIÓN}

La vieira patagónica Zygochlamys patagonica (King 1832) es un molusco bivalvo perteneciente a la Familia Pectinidae, que se distribuye a lo largo de la "Provincia Biogeográfica Magallánica", incluyendo las Islas Malvinas y Banco Burdwood (Waloszek y Waloszek 1986; Lasta et al. 1998; Bizikov y Middleton 2005). En el Océano Pacífico, las agrupaciones de esta especie se encuentran a bajas profundidades, alcanzando el límite norte de su distribución a los $42^{\circ} \mathrm{S}$ (Valladares y Andrade 1991). Por el contrario, en el Atlántico las mayores densidades se distribuyen a lo largo de la isobata de $100 \mathrm{~m}$ asociadas al frente de talud (Bogazzi et al. 2008), desde el Cabo de Hornos ( $56^{\circ} \mathrm{S}$ ) hasta Uruguay $35^{\circ} 50^{\prime} \mathrm{S}$ (Figura 1) (Riestra y Barea 2000; Gutiérrez et al. 2008).

La pesquería de vieira patagónica en aguas de la plataforma continental argentina comenzó en 1996 (Lasta y Bremec 1998). Desde que se iniciara esta pesquería, la vieira patagónica ha constituido un recurso pesquero de gran importancia comercial, ubicándose entre las primeras cuatro pesquerías marinas del país en cuanto al valor de sus exportaciones. Los desembarques de callos (músculo abductor) alcanzaron volúmenes cercanos a las $5.418 \mathrm{t}$ en 2017, siendo los principales mercados Francia, Estados Unidos y Canadá, con un precio promedio de USD 13.766 la tonelada (SSPyA 2017). Si bien existe una revisión reciente de las pesquerías de pectínidos en la Argentina (Soria et al. 2016), en este trabajo se amplía lo referente a las últimas investigaciones relacionadas con la vieira patagónica, incluyendo estimación de la eficiencia de la rastra, mortalidad residual y la descripción de los primeros estadios larvales, entre otros, trabajos de suma importancia que fueron realizados en los últimos dos años. Se presenta también una síntesis de su biología, tendencias de biomasa, historia y manejo de la pes- quería luego de 22 años de investigaciones ininterrumpidas sobre este recurso y la comunidad bentónica asociada.

\section{ASPECTOS BIOLÓGICOS Y ECOLÓGICOS}

\section{Distribución y tipo de fondo}

La población de vieira patagónica en la Argentina está estructurada en varios bancos discretos de dimensiones variables y discontinuas. A macro escala, la distribución espacial de esta especie en el Atlántico Sudoccidental coincide con la ubicación de tres sistemas frontales: el Frente de Talud (FT), el Frente de Patagonia Norte y el Frente de Patagonia Sur (Bogazzi et al. 2005). Estos frentes, áreas con fuertes gradientes de variables oceanográficas como salinidad y temperatura, constituyen importantes hábitats para la alimentación y reproducción de muchos organismos marinos, actuando como áreas de concentración de larvas pelágicas o como barreras a su dispersión. La relación de las agregaciones de vieira patagónica con dichos frentes está relacionada con la alta disponibilidad de alimento para la especie (Bogazzi et al. 2005; Mauna et al. 2008), dado que es un molusco filtrador que se alimenta principalmente de diatomeas y otros organismos del fitoplancton (Schejter et al. 2002). Bajo la influencia del FT, y a lo largo de la isobata de los $100 \mathrm{~m}$, se encuentran los bancos de vieira más rentables (Lasta y Bremec 1998; Bogazzi et al. 2005).

Los sedimentos del área del talud varían entre fango y arenas, con sectores localizados de conchillas, gravas y afloramientos rocosos (Parker et al. 1997). Por lo general, son sedimentos homogéneos, con predominio de arenas finas con importante presencia de restos de invertebrados productores de bioclastos, como por ejemplo conchillas de gasterópodos, valvas de vieira, testas de equinodermos (Bremec et al. 2014). La presencia de la vieira patagónica podría estar relacionada 


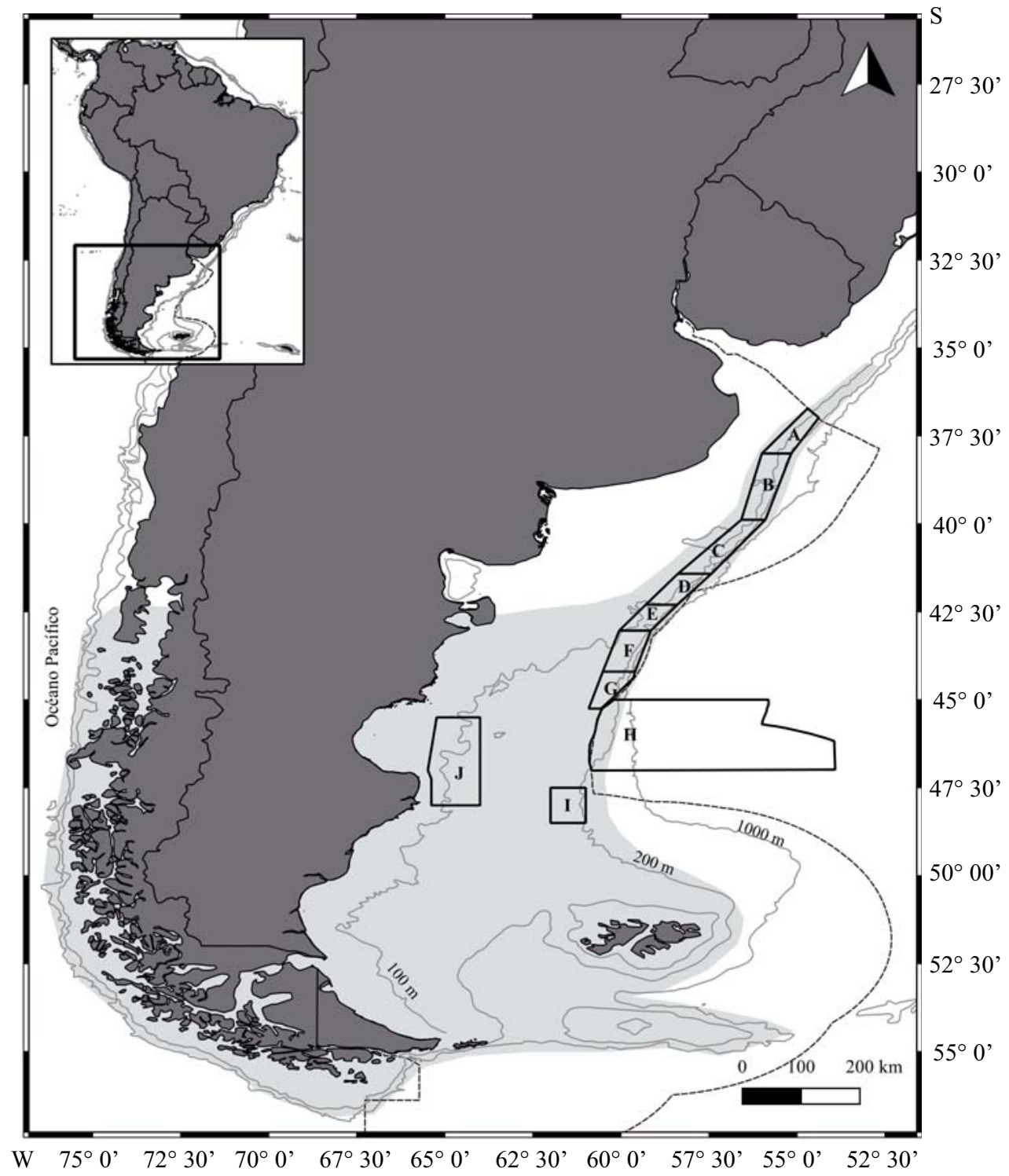

Figura 1. Distribución de las unidades de manejo (A-J) del recurso vieira patagónica (Zygochlamys patagónica) en la plataforma continental argentina.

Figure 1. Distribution of Patagonian scallop (Zygochlamys patagonica) management units (A-J) in the Argentine continental shelf.

con los sedimentos arena y arena muy fina (Madirolas et al. 2005; Lasta 2013). Si bien hasta el momento no se ha encontrado un patrón en la distribución espacial de la especie y/o su fauna acompañante en función del tipo de sedimento (Lasta 2013; Bremec et al. 2014).

\section{Reproducción, asentamiento y reclutamiento}

El ciclo reproductivo de la vieira patagónica, descripto para la Unidad de Manejo (UM) B, presenta un desarrollo sincrónico de gametas y del período de desove, con emisiones parciales duran- 
te primavera y verano (Campodónico et al. 2008). Los factores disparadores del desove se han relacionado principalmente con la disponibilidad de alimento (Campodónico et al. 2008). Si bien la temperatura es un disparador importante en el ciclo reproductivo de los pectínidos, esto no parece cumplirse para la vieira patagónica, posiblemente debido al acotado rango de temperaturas $\left(6-8^{\circ} \mathrm{C}\right)$ al que se encuentra expuesta (Lomovasky et al. 2007). Así mismo, el extenso período de desove, junto con las emisiones parciales, ha sido propuesto como una estrategia para maximizar la progenie en ambientes altamente inestables, principalmente debido a la variabilidad en la disponibilidad de alimento (Campodónico et al. 2001, 2008).

El establecimiento de nuevos bancos y el mantenimiento de los actuales dependen de la colonización exitosa de las larvas en un sustrato adecuado. Las circunstancias y factores que deben propiciarse para cumplir con ese objetivo son muchas, e involucran diferentes escalas espaciales (desde milímetros hasta cientos de kilómetros). Entre estos factores podemos citar la densidad y condición de los progenitores, el encuentro de gametas, el trasporte y supervivencia de las larvas, el asentamiento de las mismas en un sustrato adecuado y la supervivencia de los reclutas, como los más importantes (Caddy 1975; Orensanz et al. 2016).

La distribución y abundancia de los pectínidos están influenciadas por características del hábitat, tales como profundidad, tipo de sustrato, corrientes, turbidez, salinidad y disponibilidad de alimento (Brand et al. 2006). A una escala espacial más fina, los componentes estructurales del hábitat, como la presencia de tubos de poliquetos, hidrozoos, esponjas, macroalgas y/o valvas, proveen de sustratos de asentamiento para las larvas recién asentadas de vieira o "spat" (Harvey et al. 1993; Bradshaw et al. 2003; Kamenos et al. 2004). La fijación de los reclutas sobre estas estructuras reduce la tasa de depredación, mejora el crecimiento al tener acceso a comida de mejor calidad y evita la asfixia por sedimento (Brads- haw et al. 2003; Pearse et al. 2004; Howarth et al. 2011; Mendo et al. 2014).

En el caso de la vieira patagónica, se registraron reclutas recién asentados (alto total de valva (AT) entre $0,48-3,5 \mathrm{~mm}$ ) a diferentes latitudes y profundidades, principalmente sobre hidrozoos, $\mathrm{y}$ en menor medida sobre vieiras adultas, constituyendo éstos los sustratos de asentamiento primario (Bremec et al. 2008). Cabe mencionar que la sola presencia de hidrozoos no garantiza el reclutamiento de la especie, y que aún se desconoce si existe relación entre la intensidad del reclutamiento y la disponibilidad de sustratos de asentamiento.

Si bien aún no se cuenta con la descripción del desarrollo larvario completo de esta especie, de acuerdo con la relación de la temperatura con la talla de la prodiscoconcha de Cragg (2006), la larva de la vieira patagónica sería planctotrófica, y su período en la columna de agua no excedería los 74 días (Schejter et al. 2010). Schwartz y Campodónico (2019) describen las etapas iniciales del ciclo larval de la vieira patagónica en condiciones controladas, reconociendo las diferentes estructuras de los primeros estadios embriológicos hasta larva trocófora. Debido a la mortalidad total producida durante esta fase, los autores no pudieron identificar los estadios larvales siguientes. La fase larvaria trocófora es la más crítica para los pectínidos debido a que posee cuerpo desnudo (sin valvas) que la hace más vulnerable.

Actualmente, se continúa investigando a fin de determinar características de las siguientes fases (tiempo de cada estadio, tipo y forma de nado, etc.), las cuales son relevantes para confirmar el tiempo que la larva permanece en la columna de agua, y por consiguiente, su capacidad de dispersión y momento de asentamiento al sustrato. Esto constituiría un gran avance en el proceso de entender los distintos factores que condicionan e influyen en los primeros estadios de vida de esta especie.

Hasta el momento se han identificado dos reclutamientos masivos al fondo en los bancos de vieira patagónica. El primero fue estimado por retrocálculo de acuerdo con la distribución de fre- 
cuencia de tallas, y corresponde con el desove de primavera de 1994 y verano de 1995 (Valero 2002). Este evento se registró principalmente en el Banco Reclutas (actualmente UM B). El segundo reclutamiento masivo fue detectado directamente por la alta presencia de individuos menores a $15 \mathrm{~mm}$ AT en las campañas de evaluación, el cual coincidió con el período de desove 2000-2001 (Lasta et al. 2001).

El reclutamiento en esta especie parece no tener un patrón recurrente en espacio y tiempo, aunque su distribución en las UM más septentrionales está influenciada por la posición del Frente de Talud (Mauna et al. 2008). En los años de reclutamiento exitoso antes mencionados, se observó que las mayores abundancias de los reclutas se localizaron en las cercanías de la posi- ción climatológica del FT y las mayores concentraciones de clorofila $a$ (Mauna et al. 2008). Este patrón de ocurrencia de los principales reclutamientos a lo largo del FT puede ser un fenómeno complejo donde el FT limitaría el desplazamiento de las larvas, pero a su vez, permite la deriva a lo largo del mismo con el subsiguiente retorno al fondo (Shanks 1995). A pesar de que los mecanismos que explican este proceso se desarrollan a lo largo de todo el FT, desde 2001 y hasta la actualidad no se detectaron reclutamientos masivos. En la Campaña de Evaluación VA 12/2018 dirigida a la UM B se registró una importante presencia de reclutas (Figura 2), sin embargo, este evento no fue considerado un reclutamiento masivo dado que solo se registró en algunas áreas de la UM B (Campodónico et al. 2019).

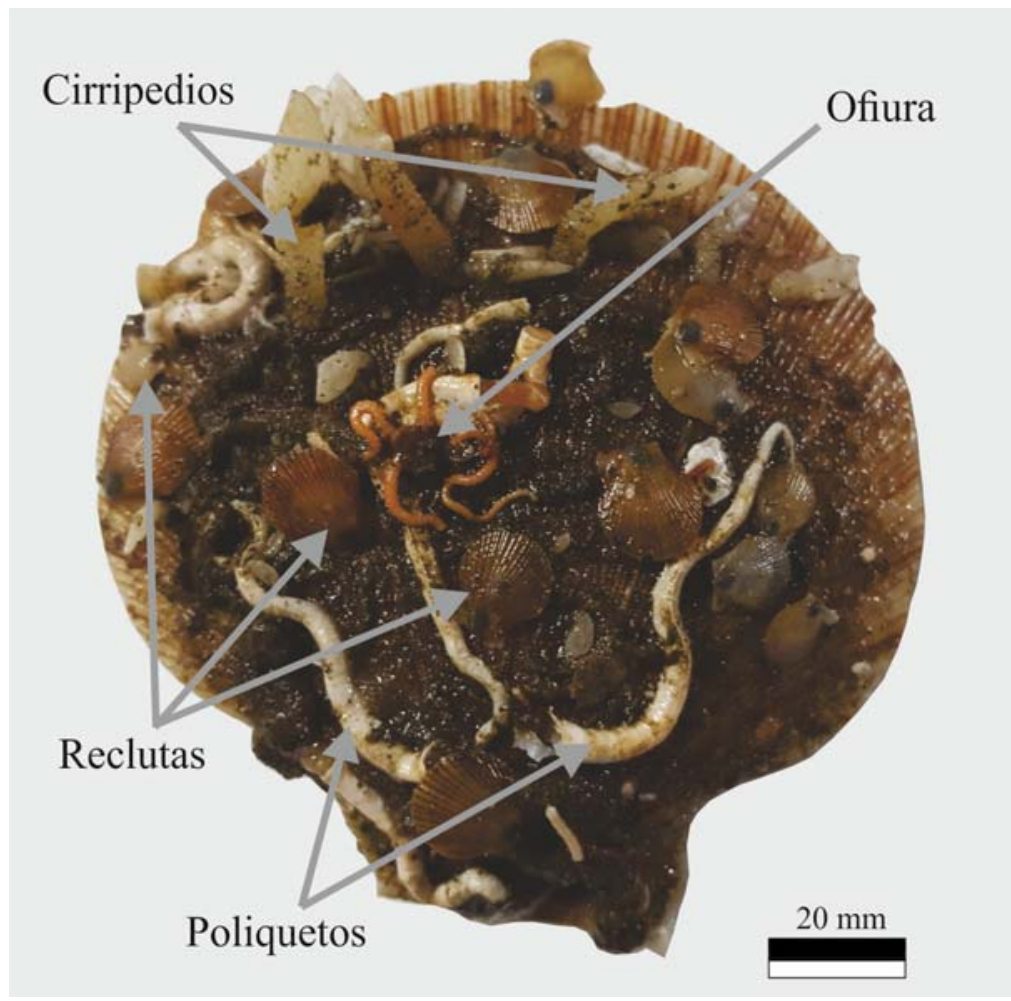

Figura 2. Juveniles de vieira patagónica (> $20 \mathrm{~mm}$ AT) asentados sobre un ejemplar adulto observado en la Unidad de Manejo B durante la Campaña de Evaluación VA 12/2018.

Figure 2. Patagonian scallop juveniles (> $20 \mathrm{~mm}$ SH) settled on an adult specimen observed in Management Unit B during the VA 12/2018 research survey. 
Si bien se sugirió un período de 5-6 años entre reclutamientos exitosos (Valero 2002), la ausencia de reclutamiento masivo en el período 20052006 y en años subsiguientes no avala esta teoría (Campodónico et al. 2017a, 2017b). En consecuencia, pese a la fuerte relación entre los frentes marinos y las concentraciones de alimento disponibles, y a la presencia de stock desovante, el reclutamiento de esta especie presenta una dinámica muy incierta y difícil de prever. Como se mencionó en párrafos anteriores, esta incertidumbre es común a muchas especies de pectínidos.

De acuerdo con Caddy y Gulland (1983), los patrones de fluctuación en los stocks pesqueros pueden clasificarse en función de los patrones de las fluctuaciones de la intensidad de los reclutamientos. De los cuatro tipos de stocks que estos autores describen, el que más se ajusta a la vieira patagónica es el de stock irregular: stocks que fluctúan ampliamente de un año a otro sin presentar un patrón claro. Tal el caso de poblaciones cuyo reclutamiento se encuentra fuertemente influenciado por las condiciones hidrográficas.

\section{Crecimiento y mortalidad}

Z. patagonica es una especie longeva, similar a otras especies de profundidad (entre 100 y 200 $\mathrm{m})$. La edad máxima alcanzada varía con la latitud, aumentando de 13 a 25 años de norte a sur (ver Lomovasky et al. 2008, 2011). La talla de primera madurez ha sido determinada a diferentes latitudes, siendo $36 \mathrm{~mm}$ AT a $39^{\circ} \mathrm{S}$ (Campodónico et al. 2008, 2009) y $45 \mathrm{~mm}$ en el rango comprendido entre los $40^{\circ} \mathrm{S}$ y $54^{\circ} \mathrm{S}$ (Waloszek y Waloszek 1986). De esta manera, la talla legal comercial de vieira patagónica $(55 \mathrm{~mm}$ AT) es alcanzada entre los 3 y 5 años de edad, dependiendo de la ubicación latitudinal, y permite al menos dos desoves consecutivos (Campodónico et al. 2008).

Las tasas de crecimiento, así como también el peso total del individuo, el peso de sus partes blandas, el peso de músculo y el peso de la góna- da varían estacional (Valero 2002; Lomovasky et al. 2007) y latitudinalmente (Defeo y Gutiérrez 2003; Lomovasky et al. 2008). Sin embargo, este patrón de crecimiento entre las diferentes partes del individuo no es completamente sincrónico (Lomovasky et al. 2008). Para la UM B (ex Banco Reclutas, $39^{\circ} \mathrm{S}-39^{\circ} 30^{\prime} \mathrm{S}$; $55^{\circ} 21^{\prime} \mathrm{W}-56^{\circ}$ $30^{\prime}$ W) las tasas máximas de crecimiento de valva se registraron durante los meses de invierno, mientras que las tasas máximas de crecimiento en músculo fueron en primavera y en gónada en otoño hasta la transición de invierno (Valero 2002; Campodónico et al. 2008; Lomovasky et al. 2008). Latitudinalmente, las diferencias en la morfología, patrones de crecimiento, edad máxima y tasas de crecimiento entre UM pueden estar relacionadas con los procesos hidrográficos que influyen en la productividad primaria y la disponibilidad de alimento (Lomovasky et al. 2011). A una escala menor, como por ejemplo dentro de un banco, las diferencias en las tasas de crecimiento entre cohortes pueden deberse a efectos densodependientes (Valero 2002).

La tasa instantánea de mortalidad natural de la viera patagónica fue estimada por diferentes métodos y en diferentes áreas. Por máxima verosimilitud y para un área de exclusión pesquera en la UM B, la tasa instantánea de mortalidad natural se encuentra entre 0,31 y 0,46 por año (Valero 2002). También se estimó por análisis bayesiano $(\mathrm{M}=0,31)$ con un intervalo de confianza entre 0,14 y 0,52 , y por distintos métodos indirectos, donde osciló entre 0,11 y 0,82 para toda la UM B (Milessi et al. 2010).

A fin de considerar la actividad de otras flotas pesqueras y su impacto en los bancos de vieira patagónica en la UM B, se estimó la mortalidad residual definida como la mortalidad natural más la ocasionada por otras pesquerías de arrastre que operan sobre el área de distribución de la especie (Aubone et al. 2018a); dicha mortalidad osciló entre 0,025 y 0,225 según el rango de AT de los individuos. Esta mortalidad residual es considerada alta y refiere a una dinámica poblacional de 
rápido vaciamiento, en caso de no producirse los reclutamientos y supervivencia adecuados (Aubone et al. 2018a).

\section{Estructura y distribución de la comunidad bentónica asociada a la pesquería}

La comunidad bentónica asociada a la pesquería de vieira patagónica es, quizás, una de las pocas comunidades sujetas a arrastre pesquero que cuenta con información previa al inicio de la actividad pesquera (Bremec y Lasta 2002). El monitoreo de esta comunidad ha sido uno de los objetivos principales del Programa Pesquerías de Moluscos Bentónicos (PPMB) del Instituto Nacional de Investigación y Desarrollo Pesquero (INIDEP), por lo que se dispone de una importante serie temporal, tanto con información obtenida por los observadores a bordo como a través de las campañas de investigación (Bremec et al. 2006; Schejter et al. 2013, 2014, 2017; Escolar et al. 2014).

La asociación de especies más conspicua está conformada por la vieira patagónica, la esponja Tedania sp., la anémona Actinostola crassicornis y los equinodermos Ophiactis asperula, Ophiacantha vivipara, Ophiura lymani, Sterechinus agassizii, Austrocidaris canaliculata, Diplasterias brandti, Ctenodiscus australis, Psolus patagonicus y Pseudocnus dubiosus leoninus (Bremec y Lasta 2002; Schejter et al. 2014, 2017). En esta comunidad, la vieira patagónica actúa también como ingeniero ecosistémico brindando sustrato de asentamiento y refugio a una gran cantidad de especies (Schejter y Bremec 2007).

En los bancos de vieira patagónica se identificaron hasta el momento 250 especies de invertebrados bentónicos (Schejter et al. 2013, 2017), muchas de ellas luego de trabajos realizados por especialistas en los diferentes grupos taxonómicos: briozoos (López Gappa y Landoni 2009), poríferos (Shejter et al. 2006), equinodermos (Escolar y Bremec 2009), hidrozoos (Genzano et al. 2009) y organismos infaunales y endobiontes (Sanchez et al. 2011; Schejter et al. 2012).
Un gran número de estas especies son filtradoras y suspensívoras, dado el hábitat donde se encuentran y el depredador tope en la comunidad de invertebrados es la estrella de mar Diplopteraster clarki. También existen numerosos depredadores secundarios que se alimentan directamente de la vieira patagónica, entre ellos se puede mencionar los caracoles Adelomelon ancilla, Odontocymbiola magellanica, Fusitriton magellanicus, y la estrella de mar Labidiaster radiosus (Botto et al. 2006).

Con respecto al efecto del arrastre en esta comunidad, se observó la disminución de organismos sésiles y frágiles, y el aumento de organismos depredadores y/o detritívoros al compararse con áreas de exclusión pesquera (Schejter et al. 2008, 2014; Escolar en evaluación), tal como sucede en la mayoría de las comunidades bentónicas sujetas a arrastres de fondo (Kaiser et al. 2000; Hinz et al. 2009). Este aumento se relacionó con el incremento en la disponibilidad de alimento, producto del descarte de los buques comerciales de vieira y de los organismos dañados por el paso de la red (Jennings et al. 2001).

El esfuerzo pesquero influye en la biomasa y distribución de muchas especies de la comunidad, ya que las áreas sujetas a mayor esfuerzo pesquero presentan los valores de biomasa más bajos (Bremec et al. 2015; Escolar en evaluación). Si bien en términos generales se registran las mismas especies desde el comienzo de la pesquería, estas variaron sus porcentajes de contribución. A modo de ejemplo, en la UM B disminuyó la contribución de vieira patagónica, Porifera, O. vivipara y F. magellanicus, en cambio aumentó la contribución del poliqueto Chaetopterus antarcticus (Escolar et al. 2019). Las áreas de exclusión pesquera y/o áreas sin actividad de la flota presentan mayor riqueza específica (Escolar et al. 2015).

Cabe mencionar que en los últimos años se observó un aumento en el porcentaje de fauna acompañante en las capturas de la flota comercial, incluso en varias UM la vieira patagónica dejó de ser la fracción dominante, lo que implica 
un cambio importante en la estructura de la comunidad bentónica en las áreas de pesca de vieira patagónica (Escolar et al. 2018). Los diferentes cierres implementados para la protección del recurso (Campodónico y Mauna 2014) contribuyeron de manera significativa a la recuperación de la comunidad bentónica, al menos en biomasa (Escolar et al. 2015). En este marco, la permanencia de áreas intangibles de pesca (reservas reproductivas y estación experimental fija) es de fundamental importancia a fin de analizar cambios en la comunidad.

El procesamiento de la captura a bordo de los buques comerciales también afecta a la comunidad bentónica asociada. Dicho procesamiento aumenta el nivel de daño en la mayoría de los organismos, incluso muchos gasterópodos y equinodermos quedan retenidos, por lo que se asume la no supervivencia de los mismos. La especie más afectada es el erizo $S$. agassizii (Escolar et al. 2017). En general, los ejemplares con menor nivel de daño presentan mayor supervivencia, excepto el caracol F. magellanicus, cuya supervivencia fue cercana al 100\% independientemente del nivel de daño observado (Schwartz et al. 2016). En el caso de los individuos de talla no comercial de vieira patagónica también se registró una importante disminución de la supervivencia a mayor nivel de daño (Schwartz et al. 2019).

\section{LA PESQUERÍA DE VIEIRA PATAGÓNICA}

\section{Historia}

En 1995 comenzó un programa cooperativo de investigación pesquera Estado-Empresa sobre la especie vieira patagónica, luego de que distintas campañas de investigación reportaran información sobre los patrones de distribución de esta especie y su potencial importancia económica (Lasta y Zampatti 1981; Waloszek y Waloszek 1986). Durante ese año se concretaron 15 campa- ñas experimentales en la plataforma continental argentina a bordo del buque pesquero (BP) "Erin Bruce", contando con un Observador o Técnico del INIDEP a bordo. La información recopilada permitió registrar siete nuevas concentraciones localizadas en la zona correspondiente al borde del talud, así como información sobre diferentes aspectos biológicos, ecológicos y pesqueros (Lasta y Bremec 1998).

A fines de 1995, el gobierno argentino a través de la Secretaría de Agricultura, Ganadería, Pesca y Alimentación (SAGPyA) y por medio de la Resolución SAGPyA No 19/1995, aprobó el proyecto pesquero para la explotación de esta especie por parte de las empresas Glaciar Pesquera S.A. y Wanchese Argentina S.A., con una flota compuesta por cuatro buques factorías, a razón de dos buques por empresa. Así, la pesquería nacional de vieira patagónica se inició formalmente en 1996, luego de los alentadores resultados biológico-pesqueros logrados mediante el mencionado, y poco frecuente, programa investigación Estado-Empresa (Lasta y Bremec 1998; Ciocco et al. 2006).

En marzo de 1996, se dictó la Resolución SAGPyA $N^{\circ} 150 / 1996$ mediante la que se estableció el marco legal para asegurar que la pesquería de vieira patagónica se desarrollara bajo un estricto asesoramiento científico. Esta resolución estableció además los principios básicos para el Plan de Manejo que fue instaurado en marzo de 1999, convirtiéndose en la única pesquería en la Argentina con un Plan de Manejo desde sus inicios.

Ese mismo año, el gobierno uruguayo autorizó a dos barcos a capturar vieira patagónica en la Zona Común de Pesca Argentino-Uruguaya (ZCPAU) establecida por el Tratado del Río de la Plata y su Frente Marítimo. Desde entonces y hasta fines de 2001, operaron cinco barcos en la ZCPAU, principalmente en el sector argentino de la misma. Durante el año 2000 se estableció una disputa entre ambos países sobre la capacidad de desplazamiento del recurso y la ubicación geográfica de los bancos. 
En 2002, y considerando que la vieira patagónica es un recurso sedentario, la Argentina aplicó el Artículo 77 de la Convención sobre el Derecho del Mar (CONVEMAR), el cual otorga la facultad al país en cuyo sector se encuentra el recurso a impedir que el otro país efectúe capturas frente al país ribereño dueño del recurso sedentario. Como consecuencia de esto, desde enero de 2003 la flota uruguaya se retiró del sector argentino de la ZCPAU.

El Consejo Federal Pesquero (CFP), como organismo responsable del manejo de las pesquerías en la Argentina, ha utilizado una estrategia de intercambio con el sector productivo, propiciando la participación de las dos empresas involucradas y estableciendo un alto nivel de cooperación entre el INIDEP y las compañías pesqueras.

\section{Medidas de manejo}

Las primeras medidas de manejo fueron trazadas desde el inicio de la pesquería en 1996 (Resolución SAGPyA N 150/1996), incorporando criterios adaptativos a través de medidas operacionales con impacto a corto y largo plazo. Algunas medidas estuvieron relacionadas con los resultados de las campañas anuales, como apertura-cierre de áreas y establecimiento de una Captura Máxima Permisible (CMP). Otras medidas implementadas fueron:

1) Dos áreas de manejo establecidas (Unidades de Manejo Norte y Sur), conteniendo varios bancos de pesca cada una.

2) Talla mínima legal fijada en $55 \mathrm{~mm}$ de AT (35 años).

3) Establecimiento de zonas de exclusión de pesca de arrastre en cada banco para protección del stock parental.

4) Establecimiento de zonas de exclusión de pesca de arrastre en cada banco para propósitos de investigación.

5) Relación mínima de juveniles: vieira comercial establecida en 1:1 para abrir un área a la pesca.

6) No existe una estación de pesca impuesta.

7) Esfuerzo pesquero fijo en cuatro (4) barcos.

8) Tasa de captura fijada en 0,4 de la biomasa total de vieiras comerciales para determinar la CMP.

9) Devolución inmediata al mar de los ejemplares menores a $55 \mathrm{~mm}$ AT, para permitir su supervivencia.

10)Creación de una Comisión Técnica para analizar y monitorear la pesquería, representando a la SAGPyA, al INIDEP y cada una de las empresas pesqueras.

En 1999 se estableció el Plan de Manejo formal mediante la Disposición SSP N ${ }^{\circ} 17 / 1999$, estructurado por 4 años, con la posibilidad de prorrogarlo un año más si la situación del recurso así lo hiciera aconsejable de acuerdo con los informes presentados por el INIDEP. En este plan se estableció que:

1) La captura se realizará a lo largo de todo el año calendario, pudiendo la Autoridad de Aplicación establecer vedas temporales o por zonas en caso que los informes científicos así lo aconsejen.

2) La CMP se determinará en forma anual, discriminándose dos unidades de manejo: Norte y Sur. La CMP se establecerá en relación a los bancos preexistentes.

3) La CMP determinada para cada año será distribuida en forma exclusiva entre las dos empresas poseedoras de los permisos de pesca.

4) Cada buque deberá disponer de 20 días anuales para tareas de investigación, las que se llevarán a cabo bajo la dirección del INIDEP.

5) Cada buque deberá contar con un Observador Científico designado por el INIDEP al menos en el $50 \%$ de las mareas comerciales.

En 2005, el CFP aprobó un nuevo Plan de Manejo de la especie (Resolución CFP N ${ }^{0} 4 / 2005$ ) con el objeto de continuar asegurando la sostenibi- 
lidad del recurso. Las medidas más novedosas, en relación a los planes de manejo anteriores fueron:

1) El CFP, con asesoramiento del INIDEP, dará anualmente las coordenadas del área que integra cada banco a efectos de delimitar los bancos considerados y sus respectivas CMP.

2) Se establece el accionar ante el descubrimiento de una nueva área de pesca.

3) La Dirección Nacional de Coordinación Pesquera (DNCP) llevará un control semanal de las capturas en cada banco para el que se estableciera una CMP, informando por escrito a cada empresa y al INIDEP cuando falte capturar el $10 \%$ de la CMP establecida. Una vez alcanzada la CMP el banco será cerrado a la pesca por ese año.

Por otro lado, y a través de la Resolución CFP $\mathrm{N}^{\mathrm{o}} 5 / 2005$, el CFP definió los únicos cuatro buques pesqueros autorizados para desarrollar esta pesquería, ratificando la Resolución SAGPyA No $150 / 1996$.

En agosto de 2006, y por recomendación del INIDEP, el CFP modifica el nombre y la delimitación geográfica de áreas de pesca preexistentes, además de incorporar áreas nuevas. Las denominadas unidades de manejo (Norte y Sur) pasaron a llamarse Sectores (Norte y Sur), y los Bancos se convirtieron en unidades de manejo (Resolución CFP N ${ }^{\circ}$ 9/2006). También se detallaron las medidas de administración como parte del plan de manejo de la especie por el plazo de cuatro años prorrogables por un año más.

En el marco del criterio de manejo adaptativo que requiere este recurso, el INIDEP remitió durante 2008 recomendaciones necesarias para complementar las medidas de manejo vigentes, a pedido del CFP. En base a esto, se derogó la Resolución CFP N ${ }^{\circ}$ 9/2006, y se dictó en su reemplazo la Resolución CFP No $4 / 2008$, la cual presentó algunas novedades:

1) En caso de no contar con información científi- ca proveniente del INIDEP para establecer la CMP en una UM, el CFP establecerá una CMP de carácter precautorio, ya que toda UM deberá tener asignada una CMP para ser habilitada a la pesca.

2) Se establece el factor de conversión en un valor de 7,14 (de peso de callo a peso total).

3) Los barcos habilitados reportarán diariamente a la DNCP la producción de callo y la UM en la que se realicen las capturas.

4) Se prohíbe la captura en áreas con predominio de ejemplares de talla no comercial. En caso de que en las capturas obtenidas durante dos días de pesca consecutivos se observe mayoría de ejemplares de talla no comercial, el buque deberá desplazarse hacia otras áreas de pesca.

5) La Comisión de Análisis y Seguimiento de la Pesquería de Vieira Patagónica estará conformada por dos representantes del INIDEP, dos representantes de la Autoridad de Aplicación y un representante de cada una de las empresas autorizadas a la captura de la especie. La Comisión tiene carácter de cuerpo asesor y se reunirá al menos una vez por trimestre.

En 2009, el CFP junto con el Ministerio de Relaciones Exteriores y Culto, estableció la UM 14 (Resolución CFP N² 2/2009) que se extiende hacia el este de la línea de $200 \mathrm{mn}$ hasta el borde exterior del margen continental argentino, entre $\operatorname{los} 45^{\circ} \mathrm{S}$ y $47^{\circ} \mathrm{S}$ (Figura 1). Esta acción fue motivada por la localización de nuevas concentraciones de vieira patagónica más allá de las $200 \mathrm{mn}$, y que constituyen posiblemente una continuidad de los bancos de la UM 10.

Con el objeto de contribuir a la sostenibilidad de la pesquería, también en 2009 se establecieron las Áreas de Exclusión de la Actividad Pesquera como Reservas Reproductivas en todas las unidades de manejo, prohibiendo la actividad de las pesquerías de arrastre de fondo en las mismas (Resolución CFP N 5/2009).

Durante 2012, a fin de lograr una mejor coincidencia entre las concentraciones del recurso y las 
UM que las contienen, se presentó una propuesta de redefinición de las UM en función de su distribución espacial. La misma planteó una modificación en las coordenadas que definen las UM y una nueva denominación. Esta propuesta se concretó en la Resolución CFP $\mathrm{N}^{\circ}$ 15/2012 (y su modificatoria Resolución CFP N 5/2014), y definió nuevas UM denominadas de la A a la J, y eliminando la UM 13 de muy escasa actividad pesquera (Figura 1).

En 2014, el CFP estableció el régimen específico de Cuotas Individuales Transferibles de Capturas (CITC) para la especie vieira patagónica, convirtiéndose en la única pesquería bentónica del país administrada bajo este régimen y distribuyendo el $45 \%$ de las CMP por UM a cada grupo empresario (Resolución CFP N ${ }^{\circ}$ 20/2014 y Resolución CFP $\left.\mathrm{N}^{\circ} 1 / 2015\right)$.

En la actualidad, las medidas de administración que rigen la pesquería de vieira patagónica son las enumeradas en las resolución CFP $\mathrm{N}^{\circ}$ 4/2008 y $\mathrm{N}^{\circ}$ 9/2016.

\section{Estimaciones de biomasa}

La biomasa de vieira patagónica por UM es estimada por el INIDEP a partir de información obtenida a través de las campañas anuales de investigación en las que se cumple con un diseño de muestreo de tipo regular (UM B) o aleatorio simple (UM D, E, F y G, Lasta et al. 2001). A partir de estas estimaciones el CFP determina la CMP en forma anual.

La información proveniente de las campañas de evaluación es utilizada para calcular la densidad absoluta de las poblaciones. Los valores de capturas obtenidos en las campañas son expandidos a valores absolutos aplicando el método de área barrida que tiene en cuenta la eficiencia del arte de pesca y el área barrida por el mismo en cada lance (Hernández et al. 2016), por lo cual es fundamental contar con una estimación precisa de estas últimas variables.

Durante el período 1998-2007, las campañas de evaluación de Z . patagonica se realizaron a bordo de los buques de investigación del INIDEP, utilizando una rastra como arte de pesca. Durante el período 2008-2012, de acuerdo con el Plan de Manejo (Resolución CFP N 4/2008), las campañas fueron desarrolladas a bordo de los buques de la flota comercial empleando la red característica de cada barco. A partir de 2013, y a fin de precisar información básica aplicada en la evaluación de biomasa, se reincorporó la rastra como arte de pesca utilizada en las campañas de evaluación a bordo de buques comerciales. Debido a estos cambios en el arte de pesca y buques empleados en las campañas de evaluación, se ha discontinuado una serie temporal que permitiría estimar un índice de abundancia para la evaluación del recurso.

El sistema de evaluación aplicado hasta el momento en la vieira patagónica estima año a año la CMP sin tener en cuenta la dinámica poblacional ni el impacto que puede tener esa captura sobre la población. La evaluación del impacto de la pesca sobre la dinámica poblacional solo puede observarse con el paso del tiempo. Actualmente, y con información disponible para la UM B, se está desarrollando un nuevo método de evaluación que propone alternativas para el manejo pesquero de la vieira patagónica. Este método considera la dinámica poblacional, y su proyección utiliza información de parámetros biológicos de la especie estableciendo reglas básicas, controlables y eficientes para promover la sostenibilidad biológica. Esto es de vital importancia dada la situación actual de la pesquería, ya que como se ha mencionado en numerosos trabajos (Campodónico et al. 2013a, 2013b, 2014a, 2014b, 2014c, 2015a, 2015b, 2016, 2017a, 2017b, 2018), en los últimos cinco años se registró una importante disminución en la biomasa de vieira patagónica en las diferentes UM (Figura 3). Tanto la biomasa total como la comercial presentan la misma tendencia decreciente, registrándose también una disminución entre ambas fracciones, lo que evidencia la falta de incorporación de individuos de talla sublegal a la población (Figura 3). 

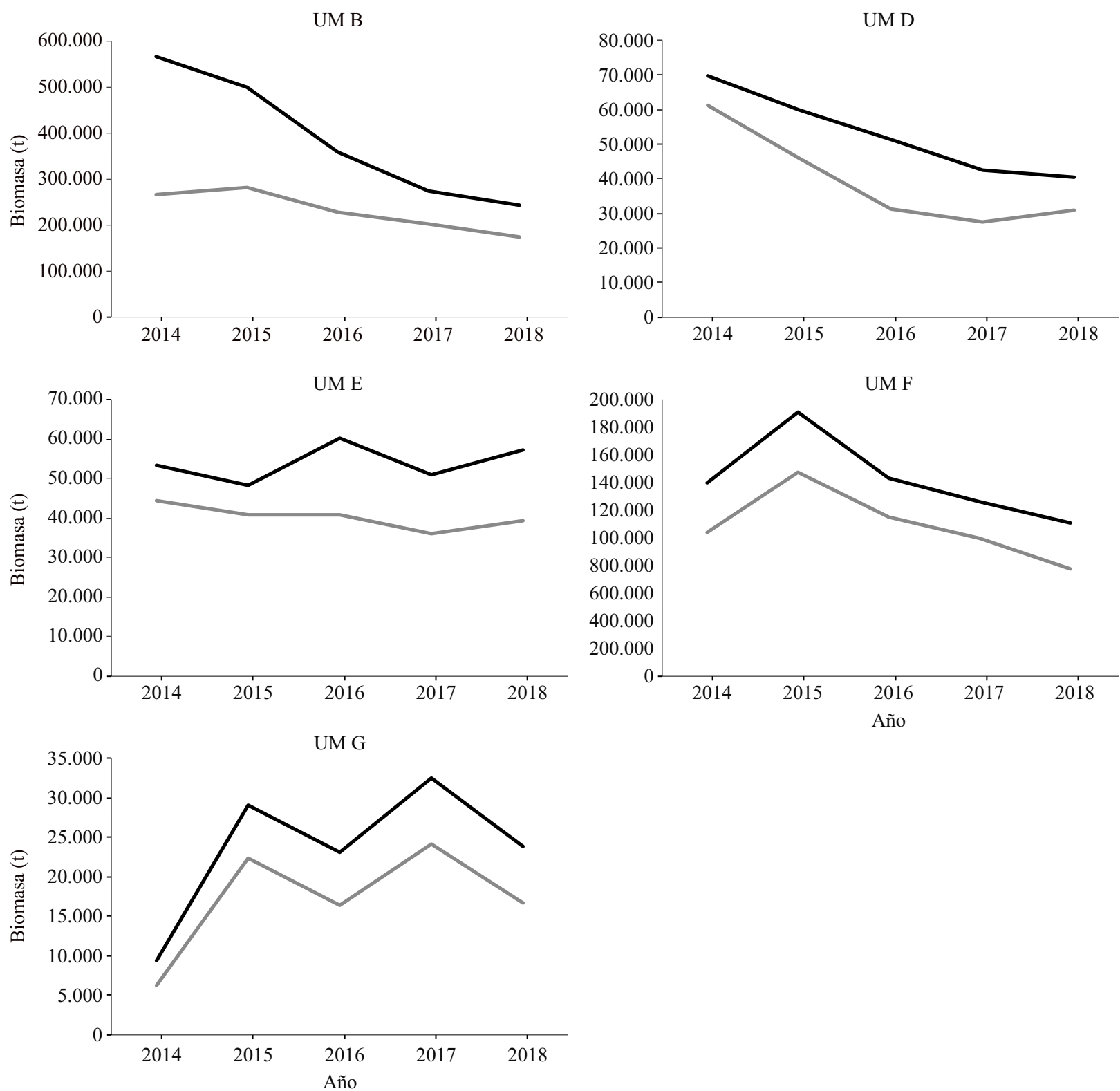

Figura 3. Evolución de la biomasa total (línea negra) y comercial (línea gris) en las unidades de manejo (UM) relevadas anualmente con campañas de evaluación de biomasa.

Figure 3. Evolution of total (black line) and commercial (gray line) biomass in the management units (UM) evaluated annualy with biomass research surveys.

\section{Artes de pesca utilizadas}

\section{Rastra-campañas de investigación}

La rastra utilizada como arte de pesca en las cam- pañas de evaluación de biomasa de vieira patagónica está conformada en su parte delantera o boca por un armazón de hierro compuesto por un tubo de 2,5 $\mathrm{m}$ de largo y $115 \mathrm{~mm}$ de diámetro unido en 
sus extremos a dos perfiles metálicos que funcionan a modo de patines, los cuales tienen contacto permanente con el fondo (Figura 4). La unión de la rastra con el cable de arrastre se realiza mediante una tijera conformada por cadenas. En la parte posterior, posee una red compuesta por dos paneles de mallas de Nylon con luz de malla de $50 \mathrm{~mm}$ y un intracopo también de nylon y luz de malla de $18 \mathrm{~mm}$. A modo de protección para el paño inferior tiene un panel de anillas de acero en la parte exterior (Roth y García 2014). Su peso en el agua es de aproximadamente $500 \mathrm{~kg}$. El valor de eficiencia de la rastra aplicado en las estimaciones de biomasa ha sido de 0,5. Este valor surgió a partir de estimaciones realizadas para un arte de pesca similar (Lasta e Iribarne 1997), generando la necesidad de obtener un valor de eficiencia empírico para la rastra utilizada actualmente en las campañas de evaluación de biomasa.

En 2018, a través de una experiencia de depleción de biomasa (Campodónico et al. 2018) se logró estimar el valor de eficiencia propio de esta rastra aplicando un método no lineal 0,676344 (Aubone et al. 2018b). En este marco, Aubone et al. (2019) presentan también una comparación de diferentes estimaciones de eficiencia aplicando diferentes métodos matemáticos, incorporando en algunos de ellos la incertidumbre en la estimación de la misma.

\section{Red comercial-pesca comercial}

Las redes (una por banda) utilizadas por la flota comercial en las mareas de pesca y en las campañas de evaluación en el período 2008-2012, poseen $22 \mathrm{~m}$ de relinga con una apertura horizontal efectiva de aproximadamente $14 \mathrm{~m}$ (Figura 4). El paño de las alas y el cuerpo es de Nylon con una luz de malla diamante de $140 \mathrm{~mm}$, mientras que el copo o bolsa es de hilo de polietileno simple con una luz de malla cuadrada de 105 y $52 \mathrm{~mm}$ de lado. A modo de protección, el vientre de la red se cubre con cabos de poliéster desflecado o parpaya (Campodónico et al. 2017c). Cabe mencionar que los diseños de las redes pueden variar entre empresas y/o buques. Esta variación se da, principalmente, en los materiales y en el tamaño y forma de las mallas (diamante o cuadrada).

Como se mencionó anteriormente, la serie temporal de datos obtenidos a partir de las campañas de evaluación se encuentra interrumpida por los cambios en la plataforma de muestreo y el arte de pesca utilizado. En 2013, con la reincorporación de la rastra en las campañas, se vuelve a disponer de datos de toda la población dado que, al utilizar las redes, ésta se encontraba sesgada hacia los individuos más grandes. Además, con la rastra también se obtiene un valor preciso de captura, contrariamente al valor obtenido en buques comerciales, donde la captura se estima visualmente. Esto trae aparejado un error de sub o sobre estimación (Aubone et al. 2016). Asimismo, se logra una correcta estimación del área barrida (Campodónico et al. 2014).

\section{Observadores a Bordo}

Un componente importante de la información biológica de las capturas en aguas argentinas surge de los datos obtenidos por el "Programa Observadores a Bordo de Buques Pesqueros" del INIDEP (Disposición SSPyA N 9/2008). Esta actividad permite obtener información temporal y espacial de todos los recursos pesqueros, la cual puede ser aplicada tanto a tareas de evaluación y monitoreo como de apoyo a la toma de decisiones en el marco de las diferentes pesquerías.

En este contexto, la pesquería de vieira patagónica es una de las pocas pesquerías en la Argentina que cuenta con un muy elevado porcentaje de viajes de pesca con Observador a bordo, alcanzando en varios años el $100 \%$ de cobertura en las mareas. Esta situación ha resultado de gran utilidad para las distintas líneas de investigación llevadas a cabo en el marco del PPMB, ya que permitió colectar una enorme cantidad de información biológica-pesquera (Escolar et al. 2009, 2018; Campodónico y Herrera 2017), incluso contribuyendo de manera decisiva 
A
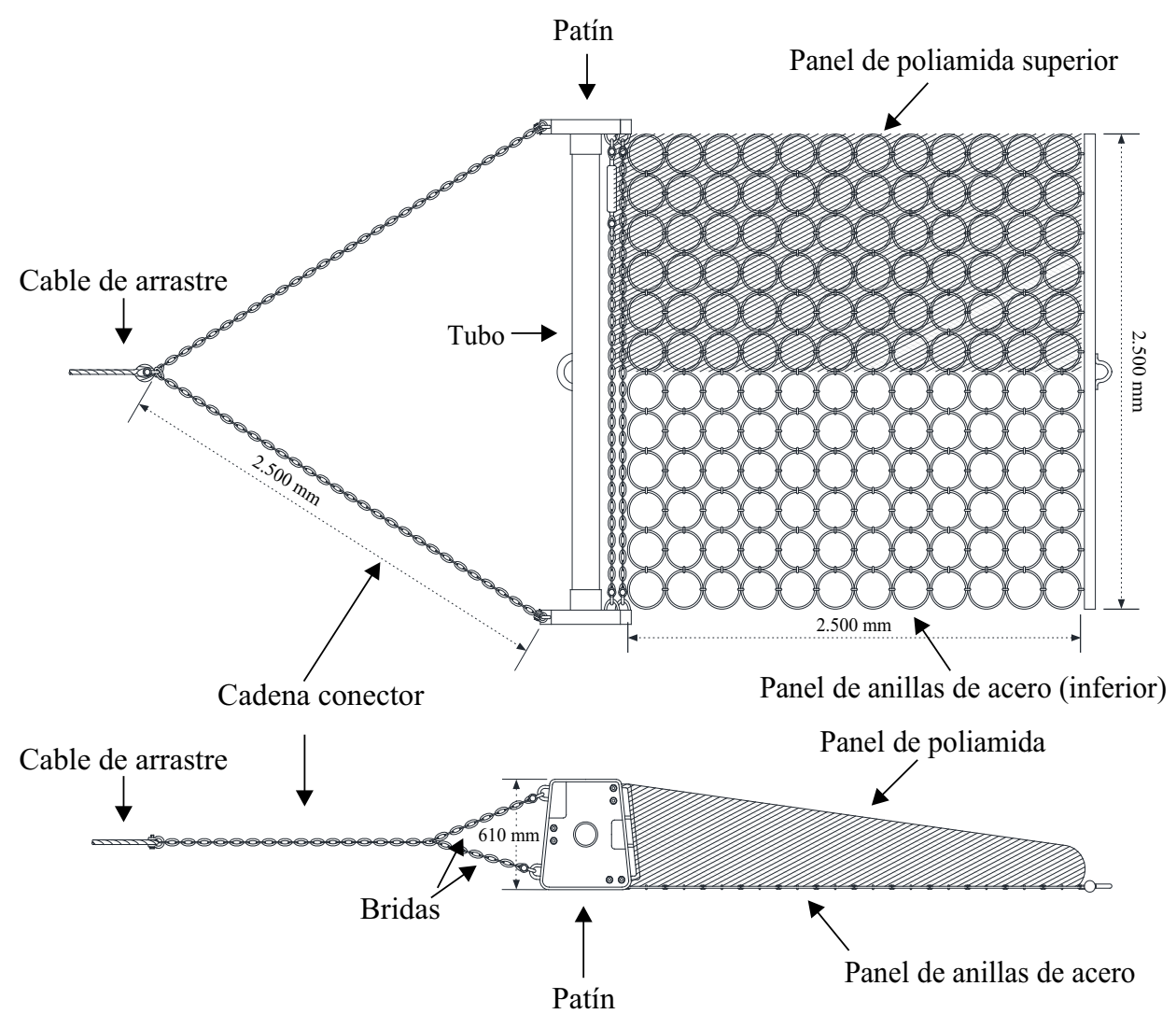

B

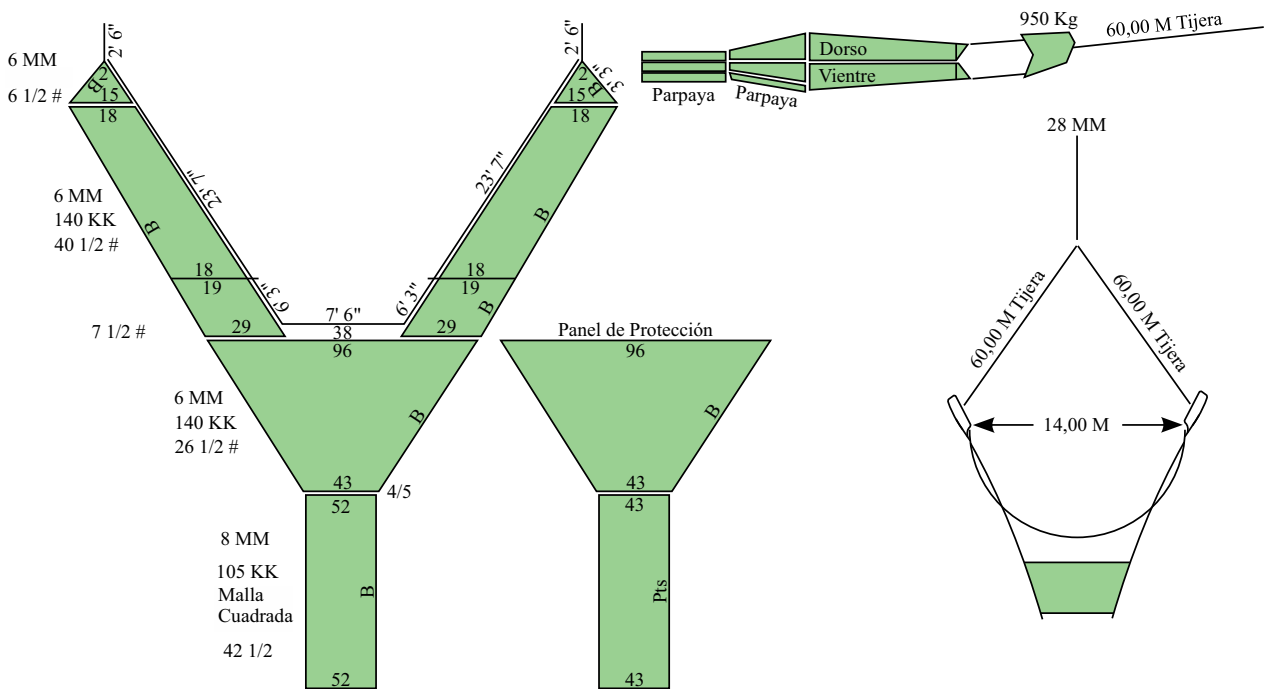

Figura 4. Arte de pesca utilizado en la evaluación de biomasa (A) y pesca comercial (B) de vieira patagónica. Figure 4. Fishing gear used in Patagonian scallop stock assessment (A) and commercial fishing (B). 
a que ésta superara con éxito los diferentes procesos de Certificación (ver más adelante).

La capacitación de los Observadores a Bordo sobre el recurso vieira patagónica está a cargo de personal Científico-Técnico perteneciente al PPMB. Mediante el dictado de clases teóricas y prácticas en el marco de los cursos que imparte el INIDEP, se instruye al Observador para realizar los distintos muestreos que requiere el protocolo de tareas (Campodónico y Escolar 2013). Luego de aprobar el examen final del curso y previo a embarcar en los buques comerciales, el Observador seleccionado y personal del mencionado programa repasan las distintas tareas a realizar a bordo (Schwartz y Herrera 2012).

En 2009, se implementó el Plan de Acción Nacional para la Conservación de Tiburones en la Argentina (PAN-Tiburones, Resolución CFP N ${ }^{\circ}$ 6/2009), estableciendo la prioridad en la adquisición de datos de condríctios en las distintas pesquerías del país. En el marco de cooperación entre Programas del INIDEP, desde 2010 el PPMB e incorporó al Protocolo de trabajo habitual una actividad orientada a registrar la presencia de rayas y tiburones en las capturas.

Posteriormente, y al regreso de las mareas, se realiza una revisión de los datos recabados a bordo por el Observador designado. Solo después de revisar, controlar, y de ser necesario corregir la información obtenida, se la incorpora a la base de datos del PPMB (Villalba, com. pers.) ${ }^{1}$.

\section{Producción y captura}

Si bien los últimos reclutamientos masivos al fondo se registraron en las temporadas reproductivas 1999-2000 y 2000-2001 a lo largo de todas las UM y con altas densidades de ejemplares AT $<15 \mathrm{~mm}$, no significa que no exista reclutamiento de ningún tipo. En años posteriores se detectaron reclutamientos localizados en distintas áreas de la distribución del recurso pero de menor importancia. La incorporación de estos individuos al stock pescable es lo que mantuvo las capturas de la última década (Figura 5). Al igual que en otras pesquerías de pectínidos, las producciones dependen fuertemente del éxito de los reclutamientos (Orensanz et al. 2016).

El esfuerzo pesquero medido como el número de redes caladas, ha variado notablemente a lo largo de los años (Figura 5), relacionado con la búsqueda, localización y densidad de las concentraciones del recurso en las distintas UM.

Las altas producciones de callo observadas entre 2005 y 2008 (Figura 5) son resultado del reclutamiento de la temporada 2000-2001, junto con las mejoras técnicas de la flota y del aprendizaje para localizar concentraciones de vieira patagónica. A partir de 2009, la flota debió invertir más tiempo en la localización del recurso debido a la disminución de áreas con gran densidad de organismos, concentrándose la actividad pesquera sobre agrupaciones menos densas. En la actualidad, la pesquería evidencia una reducción en las biomasas de captura y una limitación de las áreas factibles de pesca. La biomasa que sustenta las capturas actuales y del futuro inmediato de la pesquería se debe solo a reclutamientos localizados dentro de las distintas UM.

\section{Operatoria y comercialización}

La flota que opera sobre este recurso está compuesta por cuatro buques factoría armados para la captura y procesamiento a bordo. Actualmente, la flota cuenta con cuatro barcos (de 50 a $56 \mathrm{~m}$ de eslora) que trabajan las $24 \mathrm{~h}$ del día a lo largo de todo el año. Los buques operan con tangones y redes de arrastre de fondo completando entre $7 \mathrm{y}$ 14 mareas comerciales, cuya duración oscila entre $\operatorname{los} 20$ y 55 d, realizando de 40 a 60 lances diarios.

\footnotetext{
${ }^{1}$ Javier Villalba, "Programa Pesquerías de Moluscos Bentónicos", INIDEP, P. V. Ocampo N 1, B7602HSA, Mar del Plata, Argentina.
} 


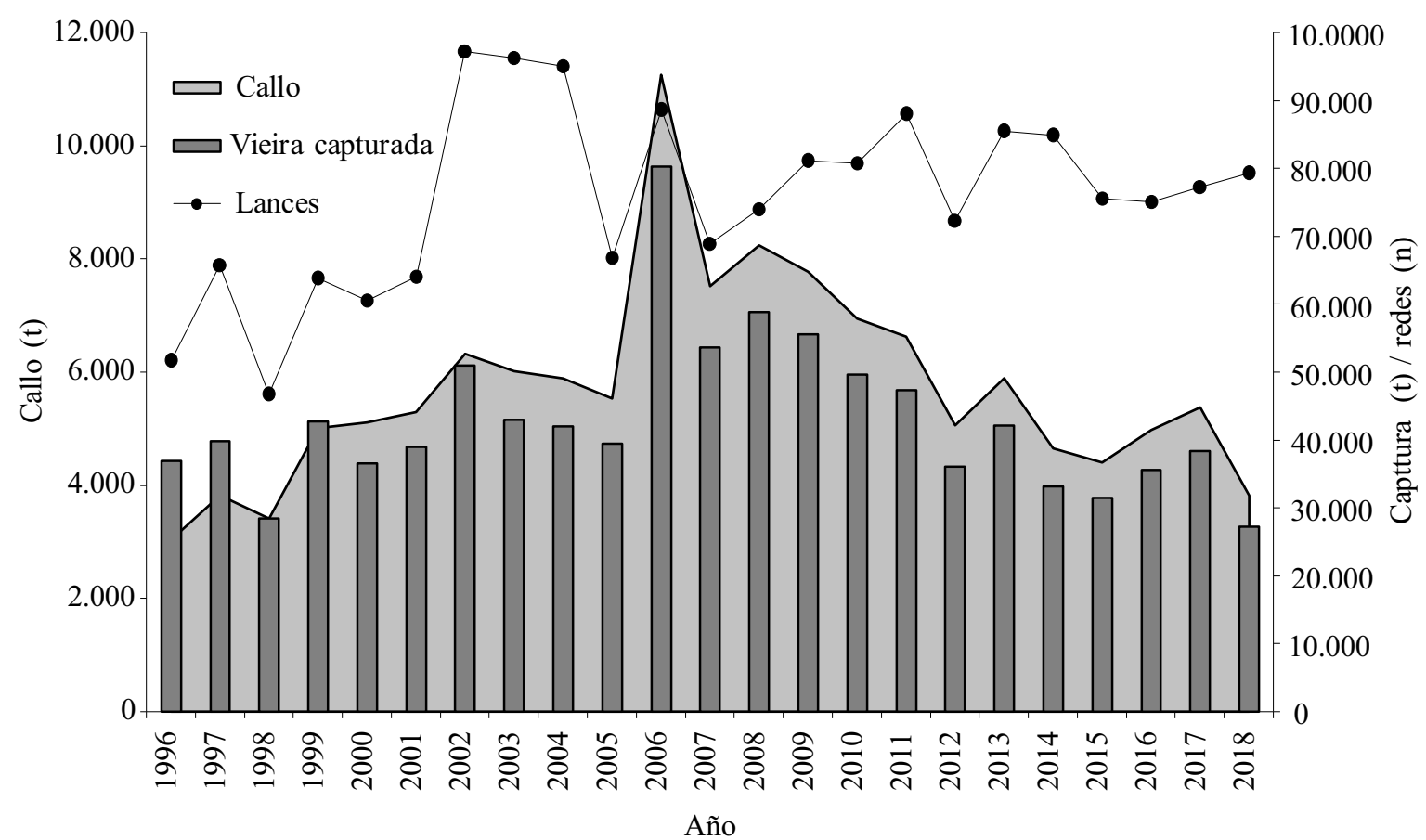

Figura 5. Capturas anuales $(\mathrm{t})$ de vieira comercial entera, esfuerzo pesquero (número de lances) y producción (callos desembarcados).

Figure 5. Annual catches (t) of whole commercial scallop, fishing effort (number of trawls) and production (meat landed).

Previo a comenzar con las tareas de pesca, se realizan lances exploratorios a fin de verificar la posible producción de las capturas en el área. Una vez determinada la zona de trabajo comienza la operatoria. El tiempo de arrastre varía entre 1 y 40 min, siendo 16 el valor promedio (Campodónico y Herrera 2017). Posteriormente al virado, las redes son abiertas y la captura descargada en la cubierta del buque y simultáneamente volcada en los pozos para luego ser transferida a la planta procesadora. En dicha planta existe una seleccionadora mecánica de tallas que descarta (y arroja al mar) la fauna acompañante y los ejemplares de vieira de talla menor a $55 \mathrm{~mm}$ AT. A las vieiras retenidas se las somete a un tratamiento con vapor de agua a fin de abrir las valvas y separarlas de las partes blandas. Posteriormente, las partes blandas son conducidas hacia máquinas denominadas "peladoras", en donde, por la acción mecánica de una serie de rodillos que giran en sentidos opuestos, se separa el músculo abductor ("callo") del resto de las partes blandas del individuo. Los restos de partes blandas y valvas son descartados al mar. Finalmente, los callos empacados y congelados se preparan para ser comercializados.

Una vez en el puerto, las cajas de producto congelado son colocadas en contenedores refrigerados pertenecientes a dos empresas líderes en el mercado de transporte marítimo. Los contenedores cargados son trasladados por vía terrestre al puerto de Buenos Aires para su posterior exportación. De acuerdo con las últimas estadísticas oficiales (SSPyA 2017), los principales mercados son Estados Unidos, Francia y Canadá (Tabla 1). En los últimos diez años el precio de exportación del producto aumentó un 44\% (Tabla 2). En promedio, los callos de vieira patagónica representan el $5 \%$ del total de las exportaciones pesqueras anuales del país. 
Tabla 1. Distribución de las exportaciones (toneladas de callo) en los principales mercados y valor promedio del producto por tonelada en 2017.

Table 1. Distribution of exports (tons of meat) in the main markets and average value (dollars) of the product per ton in 2017.

\begin{tabular}{lcc}
\hline Destino & Tonelada & Precio promedio (USD t ${ }^{-1}$ ) \\
\hline Estados Unidos & 2.469 & 12.783 \\
Francia & 2.861 & 14.558 \\
Canadá & 87 & 15.612 \\
\hline
\end{tabular}

Tabla 2. Capturas anuales (toneladas de callo) y valor promedio del producto por tonelada en el período 2007-2017. Datos obtenidos de los Informes de Coyuntura y de los de Exportaciones e Importaciones pesqueras elaborados por la SSPyA y el Ministerio de Economía de la Nación.

Table 2. Annual catches (tons of meat) and average product value per ton in the 2007-2017 period. Data derived from the Conjuncture Reports and the Export and Import Fishing Reports prepared by the SSPYA and the Ministry of Economy of the Nation.

\begin{tabular}{lcc}
\hline Año & Tonelada & Precio promedio $\left(\mathrm{USD} \mathrm{t}^{-1}\right)$ \\
\hline 2007 & 8.363 & 6.084 \\
2008 & 8.083 & 5.480 \\
2009 & 7.353 & 5.475 \\
2010 & 7.123 & 7.872 \\
2011 & 6.701 & 11.901 \\
2012 & 5.221 & 9.571 \\
2013 & 5.422 & 8.139 \\
2014 & 4.537 & 11.821 \\
2015 & 4.404 & 11.041 \\
2016 & 4.978 & 11.633 \\
2017 & 5.418 & 13.766 \\
\hline
\end{tabular}

\section{Certificación internacional de la pesquería de vieira patagónica}

El Marine Stewardship Council (MSC) es una organización sin fines de lucro, establecida en 1996 para representar los intereses de los países dedicados a la sostenibilidad a largo plazo de las pesquerías marinas y sus hábitats asociados. Su misión es la protección de la vida en los océanos reconociendo y premiando la pesca sostenible (www.msc.org).
Las directrices de MSC a los certificadores especifican que la unidad de la certificación es "la pesquería o el stock de peces combinada con el método de pesca/arte y la operatoria pesquera (actividad de los buques sobre la población pescada) así como también el marco de gestión". Los principios y criterios del MSC para la pesca sostenible son los estándares con los cuales se evaluó la pesquería. Estos se organizan en función de tres principios: El Principio 1 se refiere a la necesidad de mantener la población objetivo a un 
nivel sostenible, el Principio 2 considera la necesidad de mantener la integridad del ecosistema en el que la población objetivo existe, y el Principio 3 aborda la necesidad de un sistema de gestión eficaz de la pesca a cumplir con los requisitos del Principio 1 y 2, y garantizar el cumplimiento de regulaciones nacionales e internacionales $y$ acuerdos (ver www.msc.org).

En diciembre de 2006, promovido por una de las empresas habilitadas para la pesca de este recurso (Glaciar Pesquera S.A.), la pesquería fue eco-etiquetada como sostenible conforme a los estándares del MSC. De esta forma, la pesquería de vieira patagónica se convirtió en la primer pesquería con esta distinción en el ámbito de Sudamérica, segunda en Latinoamérica y la primer pesquería de pectínidos del mundo en lograr tal certificación, siendo el producto que se logra de primera línea en los mercados internacionales más exigentes.

Este proceso de certificación fue iniciado en noviembre de 2003. Ya en 2005, y luego de la elaboración de un informe de preevaluación, la empresa involucrada decidió pasar a la etapa de evaluación completa finalizando el proceso de certificación el 8 de diciembre de 2006, con validez por un lapso de cinco años, y la aprobación de la solicitud de certificación por el MSC. La evaluación independiente fue conducida por la certificadora Organización Internacional Agropecuaria (OIA).

En marzo de 2012, y promovido ahora por las dos empresas que operan sobre el recurso, la pesquería de vieira patagónica alcanzó un puntaje superior a 80 (sobre un total de 100) para cada uno de los tres Principios antes mencionados, logrando con éxito su primera Recertificación por parte de la OIA por un período de cinco años más. La misma se cumplió en forma exitosa alcanzando los objetivos propuestos por el equipo evaluador (Morsan et al. 2013, 2014, 2015, 2016). En la actualidad, la pesquería ha sido Recertificada por segunda vez para el período 2017-2022.

\section{CONCLUSIONES}

La pesquería de vieira patagónica en la Argentina, con un desembarque promedio anual en la última década de $6.146 \mathrm{t}$ y un valor promedio de USD 9.343 la tonelada de callo, se convirtió rápidamente en una de las pesquerías de pectínidos más importantes a nivel mundial. Es quizás, una de las más completas de la Argentina en cuanto a disponibilidad de datos biológicos-pesqueros: cuenta con una gran cantidad de información espacio-temporal, tanto del recurso como de la comunidad bentónica asociada, contemplando incluso datos previos al inicio de la pesquería. El plan de manejo adaptativo implementado y la cooperación de las empresas involucradas, permitieron que las campañas de investigación se realizaran de manera casi ininterrumpida a lo largo de la historia de la pesquería. Sin embargo, luego de 22 años de actividad pesquera, es necesaria la revisión y actualización de parámetros poblacionales, tarea que está en proceso gracias al trabajo interdisciplinario que está llevando adelante el PPMB.

Actualmente la pesquería se encuentra en un momento particular dado por la falta de reclutamiento y la disminución de biomasas en las áreas de pesca. Es por eso que, en el marco de un manejo precautorio, es de suma importancia que las medidas de administración de la pesquería se basen en la mejor información biológica-pesquera disponible, a fin de lograr la sostenibilidad biológica del recurso.

\section{AGRADECIMIENTOS}

Los autores quieren agradecer a todos los colegas que participan y/o participaron del Programa de Pesquerías Moluscos Bentónicos, y a los que, aun no perteneciendo al mismo, hicieron posible 
la realización de los trabajos mencionados en esta síntesis. También queremos agradecer a los dos revisores anónimos, quienes con sus comentarios y sugerencias ayudaron a mejorar este trabajo. Un agradecimiento especial a nuestro querido colega Daniel Hernández†. Contribución INIDEP $\mathrm{N}^{\circ}$ 2193.

\section{REFERENCIAS}

Aubone A, Campodónico S, Escolar M. 2018 a. Estimación de la mortalidad residual a la pesquería de vieira patagónica, Unidad de Manejo $\mathrm{B}$, año 2014. Inf Invest INIDEP $\mathrm{N}^{\circ}$ 69/2018. 8 p.

Aubone A, CAmpodónico S, Escolar M, GarCÍA J. 2019. Estimaciones de la eficiencia de captura de la rastra usada en evaluación de biomasa de vieira patagónica (Zygochlamys patagonica). Inf Téc Of INIDEP N ${ }^{\circ}$ 16/2019. $11 \mathrm{p}$.

Aubone A, CAmpodónico S, García J, Escolar M. 2018b. Eficiencia de captura de la rastra utilizada en evaluación de biomasa de vieira patagónica (Zygochlamys patagonica). Inf Téc Of INIDEP N 39/2018. 14 p

Bizikov VA, MidDleton DAJ. 2005. Scallop Zygochlamys patagonica (Bivalvia; Pectinidae): distribution, stock structure and fishery potential in the Falkland Islands waters. Ruthenica. 15: 23-60.

Bogazzi E, Baldoni A, Rivas A, Martos P, Reta R, Orensanz JM, Lasta M, Dell'arciprete P, WERNER F. 2005. Spatial correspondence between areas of concentration of Patagonian scallop (Zygochlamys patagonica) and frontal systems in the Southwestern Atlantic. Fish Ocean. 14: 359-376.

Botto F, Bremec C, Marecos A, Schejter L, LASTA M, IRIBARNE O. 2006. Identifying predators of the SW Atlantic Patagonian scallop Zygochlamys patagonica using stable iso- topes. Fish Res. 81: 45-50.

Bradshaw C, Collins P, Brand AR. 2003. To what extent does upright sessile epifauna affect benthic biodiversity and community composition? Mar Biol. 143: 783-791.

BRAND A. 2006. Scallop ecology: distributions and behaviour. En: SHumway SE, PARson GJ editores. Scallops: biology, ecology and aquaculture. Elsevier Science. p. 651-744.

Bremec C, Escolar M, Schejter L, Genzano G. 2008. Primary settlement substrate of scallop, Zygochlamys patagonica (King \& Broderip, 1832) (Mollusca: Pectinidae) in fishing grounds in the Argentine Sea. J Shellfish Res. 27: 273-280.

Bremec C, Lasta M. 2002. Epibenthic assemblage associated with scallop (Zygochlamys patagonica) beds in Argentina. Bull Mar Sci. 70: 89-106.

Bremec C, Schejter L, Marecos A. 2006. Riqueza específica y asociaciones faunísticas en los bancos comerciales de vieira patagónica (Zygochlamys patagonica) a lo largo del frente de talud. Período 1995-2006. Inf Téc Int INIDEP N ${ }^{\circ} 10 / 2006.52$ p.

Bremec C, Souto V, Schejter L, Giberto D, ESCOLAR M. 2014. Relaciones fauna-sedimento en bancos de vieira patagónica del frente de talud. Unidad de manejo B (OB1205). Inf Invest INIDEP $\mathrm{N}^{\circ} 7 / 2014.13 \mathrm{p}$.

CADDY JF. 1975. Spatial model for an exploited shellfish population, and its application to the Georges Bank scallop fishery. J Fish Res Board Can. 32: 1305-1328.

CADDY JF, Gulland JA. 1983. Historical patterns of fish stocks. Mar Pol. 7: 267-278.

Campodónico S, Escolar M. 2013. Protocolo de observadores de a bordo. Inf Ases Transf INIDEP $\mathrm{N}^{\circ}$ 62/2013. 18 p.

CAmpodónico S, Escolar M, Aubone A. 2017c. Estimación del poder de pesca relativo y la eficiencia relativa entre la red comercial y la rastra en la pesquería de vieira patagónica. Rev Invest Desarr. Pesq. 31: 75-84. 
CAmpodónico S, Escolar M, Hernández D. 2014a. Vieira patagónica. Evaluación de biomasa para el año 2015. Unidades de manejo UM F y G. Inf Téc Of INIDEP N 28/2014. 19 p.

Campodónico S, Escolar M, Hernández D. 2015a. Vieira patagónica. Evaluación de biomasa para el año 2016. Unidad de manejo B. Inf Téc Of INIDEP $N^{\circ}$ 25/2015. 16 p.

CAmpodónico S, Escolar M, Hernández D. 2015b. Vieira patagónica. Evaluación de biomasa para el año 2016. Unidades de manejo D y E. Inf Téc Of INIDEP N ${ }^{\circ}$ 26/2015. 20 p.

CAmpodónico S, Escolar M, Hernández D. 2016. Vieira patagónica. Evaluación de biomasa para el año 2016. Unidades de manejo $F$ y G. Inf Téc Of INIDEP N ${ }^{\circ}$ 08/2016. 18 p.

CAMPodónico S, Escolar M, Hernández D. 2017a. Vieira patagónica. Evaluación de biomasa para el año 2018. Unidad de manejo B. Inf Téc Of INIDEP N³ 36/2017. 16 p.

CAmpodónico S, Escolar M, Hernández D. 2017b. Vieira patagónica. Evaluación de biomasa para el año 2018. Unidades de manejo F y G. Inf Téc Of INIDEP N ${ }^{\circ} 32 / 2017.21$ p.

CAmpodónico S, Escolar M, Hernández D. 2018. Vieira patagónica. Evaluación de biomasa para el año 2018. Unidades de manejo D y E. Inf Téc Of INIDEP N ${ }^{\circ}$ 18/2018. 18 p.

Campodónico S, Escolar M, García J. 2019. Informe de campaña de evaluación de biomasa de vieira patagónica (Zygochlamys patagonica). Unidad de Manejo B. Año 2018. Inf Camp INIDEP N $\mathrm{N}^{\circ}$ 3/2019. 27 p.

Campodónico S, Escolar M, Mauna C, HerNÁNDEZ D. 2014b. Vieira patagónica. Evaluación de biomasa para el año 2015. Unidades de manejo D y E. Inf Téc Of INIDEP $\mathrm{N}^{\circ}$ 21/2014. 20 p.

CAmpodónico S, Herrera S. 2017. Vieira patagónica (Zygochlamys patagonica): estadísticas de la pesquería correspondientes al año 2016. Inf Téc Of INIDEP $N^{\circ}$ 16/2016. 12 p. Campodónico S, Macchi G, Christiansen E,
LASTA M. 2009. Talla de primera madurez de la vieira patagónica, Zygochlamys patagonica en la Unidad de Manejo 2 (39²4' SL-55 ${ }^{\circ} 56^{\prime}$ WL), Atlántico Sudoccidental. Inf Invest INIDEP N $\mathrm{N}^{\circ} 36 / 2009.10 \mathrm{p}$.

CAmpodónico S, Macchi G, Lasta M. 2001. Ciclo reproductivo de la vieira patagónica Zygochlamys patagonica (King \& Broderip, 1832) en el Banco Reclutas, Argentina. Inf Téc Int DNI-INIDEP N 80/2001. 15 p.

CAMPodónico S, Macchi G, Lomovasky B, LASTA M. 2008. Reproductive cycle of the Patagonian scallop Zygochlamys patagonica in the SW Atlantic. J Mar Biol Assoc UK. 88: 603-611.

Campodónico S, Mauna C. 2014. Vieira patagónica: áreas de exclusión pesquera. Inf Invest INIDEP N ${ }^{\circ}$ 29/2014. 28 p.

Campodónico S, Mauna C, Garaffo G, HerNÁNDEZ D. 2013a. Vieira patagónica (Zygochlamys patagonica) evaluación de biomasa para el año 2014. Unidades de Manejo D y E. Inf Téc Of INIDEP N $\mathrm{N}^{\circ}$ 34/2013. 20 p.

Campodónico S, Mauna C, Garaffo G, HerNÁNDEZ D. 2013b. Vieira patagónica evaluación de biomasa para el año 2014. Unidad de Manejo B. Inf Téc Of INIDEP N 33/2013. 14 p.

Campodónico S, Mauna C, Escolar M, HerNÁNDEZ D. 2014c. Vieira Patagónica evaluación de biomasa para el año 2015. Unidades de manejo UM B. Inf Téc Of INIDEP $\mathrm{N}^{\circ}$ 23/2014. 13 p.

Ciocco NF, Lasta ML, Narvarte M, Bremec C, Bogazzi E, Valero J, Orenzans JM. 2006. Argentina. En: Shumway SE, Parsons GJ, editores. Scallops: biology, ecology and aquaculture (2nd ed.). Amsterdam: Elsevier. p. 1251-1283.

CRAGG SM. 2006. Development, physiology, behaviour and ecology of scallop larvae. En: Shumway SE, Parsons GJ, editores. Scallops: biology, ecology and aquaculture (2nd ed.). Amsterdam: Elsevier. p. 45-122. 
Defeo O, Gutierrez N. 2003. Geographical patterns in growth estimates of the scallops Zygochlamys patagonica, with emphasis in Uruguayan waters. J Shellfish Res. 22: 643646.

Escolar M, Bremec C. 2009. Familia Pterasteridae (Echinodermata: Asteroidea) en bancos de vieira patagónica en el Mar Argentino [resumen]. VII Jornadas de Ciencias del Mar, Bahía Blanca, Argentina. p. 259.

Escolar M, Campodónico S, Marecos A, SCHEJTER L. 2015. Efecto del arrastre pesquero en la comunidad bentónica asociada a la vieira patagónica. Inf Invest INIDEP $\mathrm{N}^{\circ}$ 84/2015. 23 p.

Escolar M, Herrera S, CAmpodónico S. 2018. Captura incidental de invertebrados bentónicos en la pesquería de vieira patagónica. Inf Invest INIDEP $\mathrm{N}^{\circ}$ 23/2018. $21 \mathrm{p}$.

Escolar M, Marecos A, Bremec C. 2014. Análisis de la comunidad de invertebrados bentónicos asociada a la pesquería de vieira patagónica (Zygochlamys patagonica King, 1832). Datos de observadores a bordo 1997-2009. Inf Invest INIDEP $\mathrm{N}^{\circ}$ 14/2014. 17 p.

Escolar M, Schejter L, Bremec C. 2011. Bancos de Zygochlamys patagonica en el frente de talud: el efecto del esfuerzo pesquero sobre la fauna asociada [resumen]. Congreso Latinoamericano de Malacología (CLAMA), Puerto Madryn, Argentina. p. 149.

Escolar M, Schwartz M, Marecos A, Herrera S, Díaz R, Schejter L, CAMpodónico S, BreMEC C. 2017. Daño en invertebrados bentónicos en la captura incidental de la pesquería de vieira patagónica. Rev Invest Desarr Pesq. 30: 53-73.

Genzano GN, Giberto D, Schejter L, Bremec C, Meretta P. 2009. Hydroid assemblages from the Southwestern Atlantic Ocean (3442 ${ }^{\circ}$. Mar Ecol. 30: 33-43.

Gutiérrez N, Martínez A, Defeo O. 2004. Identifying environmental constraints at the edge of a species' range: scallop Psychrochlamys patagonica in the SW Atlantic Ocean. Mar Ecol Prog Ser. 353: 147-156.

Harvey M, Bourget E, Miron G. 1993. Settlement of Iceland scallop Chlamys islandica spat in response to hydroids and filamentous red algae: field observations and laboratory experiments. Mar Ecol Prog Ser. 99 (3): 283292.

Hernández D, CAmpodónico S, Escolar M. 2016. Metodología de evaluación de la biomasa de vieira patagónica a partir de los datos de campañas de investigación. Inf Invest INIDEP $\mathrm{N}^{\circ}$ 04/2016. 14 p.

Hinz H, Pietro V, Kaiser MJ. 2009. Trawl disturbance on benthic communities: chronic effects and experimental predictions. Ecol Appl. 19: 761-773.

Howarth LM, Turner AP, WoOd HL, BeuKersStewart BD. 2011. Complex habitat boosts scallop recruitment in a fully protected marine reserve. Mar Biol. 158: 1767-1780.

Jennings S, Dinmore TA, Duplisea DE, WARR KJ, LANCAster JE. 2001. Trawling disturbance can modify benthic production processes. J Anim Ecol. 70: 459-475.

Kaiser MJ, Ramsay K, Richardson CA, Spence FE, BRAND AR. 2000. Chronic fishing disturbance has changed shelf sea benthic community structure. J Anim Ecol. 69: 494-503.

Kamenos NA, Moore PG, Hall-Spencer JM. 2004. Maerl grounds provide both refuge and high growth potential for juvenile queen scallops (Aequipecten opercularis L.). J Exper Mar Biol Ecol. 313: 241-254.

LASTA M. 2013. Observaciones sobre la composición granulométrica y tipo de sedimento en los bancos de vieira patagónica (Zygochlamys patagonica). Informe Sedimentos. Patagonian Scallop (Zygochlamys patagonica) Fishery. $1^{\circ}$ Annual Audit, March 11-13, Mar del Plata.

LASTA M, BREMEC C. 1998. Zygochlamys patagonica in the Argentine Sea: a new scallop fishery. J Shellfish Res. 17: 103-111.

Lasta M, Hernández D, Bogazzi E, Campodó- 
NICO S. 2001. Vieira patagónica, Unidad Norte de Manejo-CTMFM, evaluación de biomasa año 2001. Inf Téc INIDEP $N^{o}$ 9/2001. 25 p.

LAsta M, IrIBARne O. 1997. Southwestern Atlantic scallop (Zygochlamys patagonica) fishery: assessment of gear efficiency through a depletion experiment. J Shellfish Res. 16: 59-62.

LASTA M, ZAMPATTI E. 1981. Distribución de capturas de moluscos bivalvos de importancia comercial en el Mar Argentino. Resultados de las campañas de los B/I "Walther Herwig" y “Shinkai Maru”, años 1978 y 1979. En: ANGELESCU V, editor. Campañas de investigación pesquera realizadas en el Mar Argentino por los B/I "Shinkai Maru" y "Walther Herwig" y el B/P "Marburg”, Años 1978 y 1979. Resultados de la parte argentina. Contrib Inst Nac Invest Desarr Pesq (Mar del Plata). 383: 128130.

Lomovasky Bu, Baldoni A, Ribeiro P, Alvarez G, Lasta M, CAmpodónico S, Iribarne O. 2011. Exploring the causes of differences in growth rate of the Patagonian scallop Zygochlamys patagonica along its commercial bed distribution in the SW Atlantic. J Sea Res. 66: 162-171.

LOMOVASKY BJ, BREY T, BALdONI A, LASTA M, MACKenSEN A, CAMPodónico S, IRIBARNe O. 2007. Annual shell growth increment formation in the deep-water Patagonian scallop Zygochlamys patagonica. J Shellfish Res. 26: 1055-1063.

LOMOVASKY B, LASTA M, VALIÑAS M, BRUSCHETTI M, RiBEIRo P, CAMPODÓNICO S, IRIBARNE O. 2008. Differences in shell morphology and internal growth pattern of the Patagonian scallop Zygochlamys patagonica in the four main beds across their SW Atlantic distribution range. Fish Res. 89: 266-275.

LÓPEZ GAPPA J, LANDONI NA. 2009. Space utilization patterns of bryozoans on the Patagonian scallop Psychrochlamys patagonica. Sci Mar. 73: 161-171.
Madirolas A, Lasta M, Tripode M, Alvarez Colombo G, Campodónico S, Cabreira A. 2005. Experiencias con el ecosonda multihaz SIMRAD EM1002 instalada a bordo del BIP Cap. Oca Balda (período 2004-2005): estudios sobre el hábitat de la vieira patagónica. Inf Téc INIDEP No 84/2005. 9 p.

Mauna C, Franco B, Baldoni A, Acha M, LASTA M, IRIBARNE O. 2008. Cross-front variations in adult abundance and recruitment of Patagonian scallop (Zygochlamys patagonica) at the SW Atlantic Shelf Break Front. ICES J Mar Sci. 65: 1184-1190.

Mendo T, Lyle JL, Moltschaniwskyj NA, TraCEY ST, SEMmEns JM. 2014. Habitat characteristics predicting distribution and abundance patterns of scallops in D'Entrecasteaux Channel, Tasmania. Plos One. 9 (1): e85895.

Milessi AC, Lasta M, Iribarne O, Kittlein M. 2010. Direct and indirect estimates of natural mortality for the Patagonian scallop Zygochlamys patagonica. J Shellfish Res. 29: 381386.

Morsan E, Prenski LB, Cranfield HJ, Bridi RJ, MedinA Foucher CA. 2013. First surveillance report. Assessment against MSC principles and criteria for: Patagonian scallop fishery (Zygochlamys patagonica). 54 p. https:// fisheries.msc.org/en/fisheries/patagonianscallop-zygochlamys-patagonica-bottomotter-trawl-fishery/@@assessments.

Morsan E, Prenski LB, Medina Foucher CA. 2014. Second surveillance report. Assessment against MSC principles and criteria for: Patagonian scallop fishery (Zygochlamys patagonica). 37 p. https://fisheries.msc.org/en/ fisheries/patagonian-scallop-zygochlamyspatagonica-bottom-otter-trawl-fishery/@@ assessments.

Morsan E, Prenski LB, Medina Foucher CA. 2015. Third surveillance report. Assessment against MSC principles and criteria for: Patagonian scallop fishery (Zygochlamys patagonica). 89 p. https://fisheries.msc.org/en/ 
fisheries/patagonian-scallop-zygochlamyspatagonica-bottom-otter-trawl-fishery/@@ assessments.

Morsan E, Prenski LB, Medina Foucher CA, LACO ML. 2016. Fourth surveillance report. Assessment against MSC principles and criteria for: Patagonian scallop fishery (Zygochlamys patagonica). 97 p. https://fisheries. $\mathrm{msc}$. org/en/fisheries/patagonian-scallopzygochlamys-patagonica-bottom-otter-trawlfishery/@@assessments.

Parker G, Paterlini MC, Violante RA. 1997. El fondo marino. En: BosCHI EE, editor. El Mar Argentino y sus recursos pesqueros. Tomo 1. Antecedentes históricos de las exploraciones en el mar y las características ambientales. Mar del Plata: Instituto Nacional de Investigación y Desarrollo Pesquero (INIDEP). p. 65-87.

Pearse JS, Lockhart SJ. 2004. Reproduction in cold water: paradigm changes in the 20th century and a role for cidaroid sea urchins. DeepSea Res (II Top Stud Oceanogr). 51: 1533-1549.

RiEstra G, BAREA L. 2000. La pesca exploratoria de la vieira Zygochlamys patagonica en aguas uruguayas. En: Rey M, editor. Recursos Pesqueros no Tradicionales: Moluscos Bentónicos Marinos. Montevideo: Inf Téc INAPE/ PNUD. p. 145-152.

Roth R, Garcia J. 2014. Análisis dinámico-teórico de la relación entre la longitud del cable de arrastre y la profundidad de pesca para una rastra destinada a la captura de vieira. Inf Ases Transf INIDEP $\mathrm{N}^{\circ}$ 124/2014. 12 p.

SÁnchez MA, Giberto D, Schejter L, BremeC C. 2011. The Patagonian scallop fishing grounds in shelf break frontal areas: the nonassessed benthic fraction. Lat Am J Aquat Res. 39: 167-171.

SCHEJTER L, BREMEC C. 2007. Benthic richness in the Argentine continental shelf: the role of Zygochlamys patagonica (Mollusca: Bivalvia: Pectinidae) as settlement substrate. J Mar Biol Assoc UK. 87: 917-925.

Schejter L, Bremec C, Akselman R, Hernán-
DEZ D, SpIVAK E. 2002. Annual feeding cycle of the Patagonian scallop Zygochlamys patagonica (King and Broderip, 1832) in Reclutas bed $\left(39^{\circ} \mathrm{S}-55^{\circ} \mathrm{W}\right)$, Argentine Sea. J Shellfish Res. 21: 549-555.

SChejter L, Bremec Cs, Escolar M, Giberto D. 2017. Plataforma externa y talud continental. En: Bremec CS, Giberto D, editores. Comunidades bentónicas en regiones de interés pesquero en Argentina. Mar del Plata: Instituto Nacional de Investigación y Desarrollo Pesquero (INIDEP). p. 57-75.

Schejter L, Bremec C, Hernández D. 2008. Comparison between disturbed and undisturbed areas of the Patagonian scallop (Zygochlamys patagonica) fishing ground "Reclutas" in the Argentine Sea. J Sea Res. 60: 193-200.

Schejter L, Bremec B, Waloszek D, Escolar M. 2010. Recently settled stages and larval developmental mode of the bivalves Zygochlamys patagonica and Hiatella meridionalis in the argentine sea. J Shellfish Res. 29: 63-67.

Schejter L, Calcinai B, Cerrano C, Bertolino M, Pansini M, Giberto D, Bremec C. 2006. Porifera from the Argentine Sea: Diversity in Patagonian scallop beds. Ital J Zool. 73: 373385.

Schejter L, Chiesa IL, Doti BL, Bremec C. 2012. Mycale (Aegogropila) magellanica (Porifera: Demospongiae) in the southwestern Atlantic Ocean: endobiotic fauna and new distributional information. Sci Mar. 76: 753-761.

SCHEJTER L, Escolar M. 2017. Comparación del uso de red de arrastre comercial vs. rastra en el monitoreo de la fauna acompañante en la pesquería de la vieira patagónica (Zygochlamys patagonica) en Argentina. Rev Invest Desarr Pesq. 30: 75-87.

Schejter L, Escolar M, Marecos A, Bremec C. 2013. Seventeen years assessing biodiversity at Zygochlamys patagonica fishing grounds in the shelf break system, Argentina. Libro de 
resúmenes del 19th International Pectinid Workshop. Florianopolis, Brasil. p. 46-47.

Schejter L, Marecos Á, Bremec C. 2014. La comunidad de invertebrados bentónicos en la "Unidad de Manejo B" de la pesquería de vieira patagónica: comparación entre el área de reserva y el área de pesca a partir de datos obtenidos en el 2013. Inf Invest INIDEP $\mathrm{N}^{\mathrm{o}}$ 58/2104. $11 \mathrm{p}$.

Schwartz M, CAmpodónico S. 2019. Primera descripción del desarrollo larval temprano de la vieira patagónica (Zygochlamys patagonica). Mar Fish Sci. 32 (2): 115-124.

Schwartz M, Escolar M, Marecos Á, CAMPODÓNICO S. 2016. Supervivencia de invertebrados bentónicos capturados incidentalmente en la pesquería de vieira patagónica. Inf Téc INIDEP N ${ }^{\circ}$ 95/2016. 24 p.

Schwartz M, Escolar M, Marecos A, Herrera S, Díaz R, Villalba J, CAmpodónico S. 2019. Supervivencia de vieira patagónica de talla no comercial descartada por la pesquería. Mar Fish Sci. 32 (2): 149-157.

Schwartz JM, Herrera SN. 2012. Desempeño de los observadores embarcados en buques de vieira durante el año 2011. Inf Invest INIDEP $\mathrm{N}^{\circ} 40 / 2012.6 \mathrm{p}$.

SHANKS A. 1995. Mechanisms of cross-shelf dispersal of larval invertebrates and fish. En: MC EDWARD L, editor. Ecology of Marine Invertebrate Larvae. Florida: CRC Press. 480 p.

Soria G, Orensanz JM (Lobo), Morsán EM, Parma AM, Amoroso RO. 2016. Scallops biology, fisheries, and management in Argentina. En: Shumway SE, PARsons GJ, editors. Scallops: biology, ecology, aquaculture, and fisheries (3ed. ed.). Oxford: Elsevier Science. p. 1019-1046.

[SSPyA] Subsecretaria de Pesca y AcuiculTURA. 2017. Exportaciones e importaciones pesqueras. Ministerio de Agricultura, Ganadería y Pesca. Argentina. 43 p.

VALero J. 2002. Analysis of temporal and spatial variation in growth and natural mortality estimation with an Integrated Dynamic Model in the Patagonian scallop (Zygochlamys patagonica) [MSc thesis]. Seattle: School of Aquatic and Fishery Sciences, University of Washington. $154 \mathrm{p}$.

Valladares C, ANDrade S. 1991. Estado actual de los bancos naturales de ostiones en los fiordos Brookes y S Palma. En: ANDRADE et al., editores. Estudios repoblamiento ostión del sur en XIIa Región de Magallanes, Chile. Capítulo 1. 28 p.

Waloszek D, WaloszeK G. 1986. Ergebnisse der Forschungsreisen des FFS "Walther Herwig" nach Südamerika, LXV. Vorkommen, Reproduktion, Wachstum und mogliche Nutzbakeit von Chlamys patagonica (King \& Broderip, 1832) (Bivalvia, Pectinidae) auf dem Schelf von Argentinien. Arch Fish Wiss. 37: 69-99.

Recibido: 09-10-2018

Aceptado: 10-10-2019 